(Aus dem deutschen physiologischen Institut in Prag.)

\title{
Ueber die Wirkung von Aether, Chloroform und Alkohol auf das Leitungsvermögen motorischer und sensibler Nervenfasern des Frosches.
}

\author{
Von \\ Dr. H. Pereles und Dr. M. Sachs.
}

Mit einer Figur.

Spielmann und L u chsinger 1 ) haben gezeigt, wie durch locale Narcotisirnng eines Nerven die Leitungsfähigkeit an der Applicationsstelle vorïbergehend zum Verschwinden gebracht werden könne. Es erhob sich nun die Frage, ob dabei die sensiblen und motorisehen Fasern in gleicher Weise von den Narcoticum beeinflusst werden; bezw. welche Faserart zunächst die Leitungsfähigkeit einbiisst.

Wir versuchten dies mit Hilfe von Methoden zu entseheiden, welche den Grad der Einwirkung der angewandten Gifte auf sensible und motorische Fasern getrennt bestimmen lassen. Zur Narkose benutzten wir insbesondere Aether, daneben auch Chloroform und Alcohol.

Zunächst versuchten wir im Auftreten des gekreuzten Reflexes ein Criterium dafür zu erlangen, welche Faserart früher narcotisirt wird. Der Gedankengang, der uns hiebei leitete, war folgender: es sei eine $6 \mathrm{~mm}$ lange Strecke des blossgelegten rechten Ischiadicus des Frosches der Einwirkung des Aethers ausgesetzt; gäbe es nun einen Zeitraum, in welchem die sensiblen Fasern bereits gelähmt, die motorischen aber noch leitungsfähig sind so miisste jetzt durch Reizung der nervösen Endapparate der rechten Pfote kein Reflex mehr auszulösen sein, während bei Reizung der linken Pfote Bewegung des rechten Beines erfolgen könnte; das Gegentheil, nämlich das frühere Ausbleiben des. Reflexes auf

1) Dieses Archiv Bd. 24. S. 347. 1881. 
Ueber die Wirkung von Aether, Chloroform und Alkohol etc.

das rechte Beiu bei Reiznng der linken Pfote, müsste eintreten, wenn die motorischen Fasern früher als die sensiblen gelähmt werden. Diese Versuchsweise führte nicht zu verwendbaren Ergebnissen, weil sich die Auslösung des gekreuzten Reflexes nicht genïgend beherrschen liess.

Um nun mit Hilfe des gleichseitigen Reflexes dic vorliegende Frage zu entscheiden, wählten wir folgende einfache Versuchsanordnung:

Bei einem grösseren Frosch wurde unter Schonung der Blutgefässe der Ischiadicns frei präparirt. Die Schonung der BIntgefässe ist desshalb zweckmitssig, weil sonst die sensiblen Endapparate in ihrer Erregbarkeit verändert werden kïnnten. Um die Aethernarcose streng local appliciren zu können, trafen wir folgende Einrichtung: Wir nahmen eine Kantschukröhre von ca. $5 \mathrm{~mm}$ Durchmesser und schlugen mit einem feinen Locheisen durch das plattgedruickte Rohr. Die so entstandenen Oeffnungen in der Wand des Kautschnkrohres, die einander gegeniberliegen, wnrden mittelst eines queren Schnittes dureh dic halbe Röhrenwand verbunden. Hatte min den Nerv in den dureh Krümmung des Rohres klaffend gemachten spalt eingesenkt, so konnte man dureh Geradstreekung des Rohres die spaltlippen wieder selliessen, während der Nerv entsprechend den ausgelochten Stellen za liegen kam, so dass jeder Druck auf den das Röhrehen durehziehenden Nerven vermieden war. Anderseits schloss die Wand des Röhrchens an der Ein- und Anstrittsstelle des Nerven eng genug an, um ein crhebliches Uebergreifen der durch das Rohr streichenden Actherlimple auf den ausserhalb des Röhrchens gelegenen Nerv zu verhindern. Das Röhrehen wurde nun einerseits in Verbindung gesetzt mit dem kiurzeren liohr einer mit Aether (resp. Alcohol oler Chloroform) gefüllten sinitzflasehe, deren längerer Schenkel mit einem Kautschukgebläse verbunden war, so dass man nach Belieben in einzelnen Stiissen mit Aetherdampf gemiselite Luft durch das von Nerven durchsetzte Kantschukrohr leiten konnte, olne dass die ausserhally des Rolures befindlichen Theile des Nerven oder das Thier überhanpt nit dem Aetherdampfe in Beriburng kanen. Durch eine verschliessbare Abzweigung war anch Vorsorge getroffen, dass man jederzeit mit der Zufulur von Aether. dimpfen anfhören und statt derselben fenchte Luft uber das eingeschlossene Nervenstiick streichen lassen konnte. 
Als Hautreiz zur Hervorrufung der Reflexbewegung wurden ebenso wie zur directen Reizung des Ischiadicusstammes Inductionsströme verwendet, welche durch Platindrähte zugeleitet wurden. Nachdem der Ischiadicus unterhalb des Abganges der Oberschenkeläste in das Kautschukröhrchen gelegt worden war, bestimmten wir zunächst den Minimalreiz, der oberhalb der zu narcotisirenden Strecke auf den Nerven wirken musste, um eine Bewegung der Pfote hervorzurufen; ferner wurde der ungefähre Minimalreiz gesucht, der erforderlich war, um von einer beliebigen Stelle der Sehwimmhant aus mit Sicherheit eine reflectorische Bewegung des betreffenden Beines anszulösen. Hierauf wurde mit der Narcotisirung der eingeschlossenen Nervenstrecke begonnen. Es zeigte sich regelmässig, dass unter Anwendung der erwähnten Minimalreize die von der Pfote ausgelöste Reflexbewegung früber schwand als die Bewegung auf Reizung des Ischiadicus oberhalb der narcotisirten Strecke. Man konnte sogar weit über den Minimalreiz gehen, ohne Reflexzuckung von der Pfote aus zu erhalten, während fiir die directe Reizung des Ischiadicus oberhalb der narcotisirten Stelle der Minimalreiz noch geniugte, IIm Bewegung der Pfote hervorzurufen. Bald nachdem die reflectoriscbe Bewegung versehwunden war, blieb auch die Bewegung auf Reizung des Stammes aus; auch hier konnte daun der anfängliche Minimalreiz weit überschritten werden, ohne dass wir Zuckung erhielten. Dass dies Folge der durch die Narense bewirkten localen Leitungsunterbrechung war, liess sich daraus erweisen, dass Reizung unterhalb der narcotisirten Strecke Bewegung zur Folge hatte und dass wir durch Luftzufuhr die Narcose beseitigen und damit die Wirksamkeit beider Minimalreize wiederherstellen konnten. Letzteres bewies zugleich, dass die zuvor bestandene Unterbrechung der Nervenleitung nicht die Folge eines Druckes auf den Nerven war.

Wir erblickten in den Resultaten dieser Versuche nicht mehr als einen Wahrscheinlichkeitsbeweis für eine früher eintretende Narcose der sensiblen Nerven. Das von uns beobachtete frihere Ausbleiben der reflectorischen Bewegung liess sich nämlich noch anders erklüren als durch die allgemeine Annahme, dass bei Narcotisirung des gemischtfaserigen Froschischiadicus die sensiblen Fasern ihre Leitungstahigkeit fruher einbuissen als die motorischen. Die Art, wie wir den Aether auf den Ischiadicusstamm einwirken liessen, schloss nicht ein ungleichmässiges Fortschreiten der Nar- 
cose im Gesammtquerschnitte des Nerven aus. Ferner kommt in Betracht, dass wir die Prïfung auf das Erhaltensein der Leitungsfähigkeit bei den motorischen Fasern auf die Gesammtheit derselben, bei den sensiblen Fasern aber nur auf einen winzigen Bruchtheil der im Ischiadicus verlaufenden centripetalen Fasern erstreckt hatten. Unserer Versuchsanordnung haften ferner die Fehler an, dass der Erfolg bei Reizung der sensiblen Nervenendigungen zur Bedingung hat, dass die Erregung die narcotisirte Stelle zweimal durchsetzt hat, und dass die sozusagen natuirlichere Reizung der Nervenenden der kiinstlichen Reizung des Nervenstammes nicht äquivalent ist. Hieraus liesse sich vielleicht das spätere Schwinden der Bewegung bei directer Reizung des Ischiadicns erklären. Uebrigens konnte man bei dieser Versuchsanordnung von vorneherein nur dann ein positives Resultat erwarten, wenn die Erregbarkeit der sensiblen Fasern friher erlischt, als die der motorischen.

Die angeführten Mängel suchten wir durch folgende Versuchsanordnung $\mathrm{zu}$ beseitigen. Wir präparirten an einem grossen Froseh den Ischiadicus einer Seite in der Weise frei, dass nach Entfernung des gauzen Oberschenkels der Unterschenkel und der Leib nur noch durch den Nervenstamm verbunden waren; hierauf wurde das Thier an den vorderen Extremitäten auf ein Präparirbrett gebunden, die Mitte der freigelegten Nervenstrecke in das oben beschriebene Röhrehen gelegt. Oberhalb und unterhalb der zu narcotisirenden Strecke wurde je ein Paar Platinelectroden, welche mit je einem Inductionsapparat in Verbindung waren, danernd angelegt. Um wirksame Stromschleifen oder unipolare Ableitungen zu verbindern, wurden von Prof. H erin g construirte Electroden bentitat, wie sie schon längere Zeit im hiesigen Institut in Verwendung stehen. Die Figur stellt diese Electroden schematisch und vergrössert dar. Der Nerv wird quer über vier parallel and

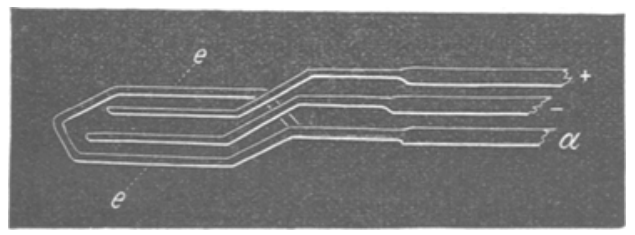

nahezn in einer Ebene gelegene Platindrähte gebriickt. Die beiden innern sind die mit den Enden der secundären Spirale verbundenen 
Electroden; die beiden äussern (e, e) sind unter sich leitend verbunden und von einem isolirenden Stabe a gehalten. Die von den Electroden extrapolar abfliessenden Electricitäten gleichen sich durch die äusseren Drähte ab. Dass dabei die sozusagen extrapolar zwischen je einer Electrode und einem äusseren Drabt liegenden kurzen Nervenstrecken mit erregt werden, ist bier, wie in den meisten Fi:llen gleichgiltig ${ }^{1}$ ).

Die Versuche wurden sowohl an geköpften als auch ungeköpften Thieren angestellt, wobei sich kein wesentlicher Unterschied zeigte. Reizt man an einer der beiden Stellen gentagend stark, so erfolgt ausser einer Bewegung der Pfote auch allgemeine Unruhe des Körpers als Folge der Erregung der sensiblen Nervenfasern. Die Bewegungen der Pfote konnten stets durch schwächere Reize ausgelöst werden als die Bewegungen des ganzen Körpers. Die Stärke der Reize wurde nun so gewählt, dass bei Reizung unterbalb der zu narcotisirenden Stelle ausser der Bewegung der Pfote eben noch deutliche Unruhe des Körpers eintrat, bei Reizung oberhalb jener Stelle nur Bewegung der Pfote, manchmal auch eine ganz schwache Bewegung des Körpers erfolgte, Dieser letztere Reiz war demnach stets kleiner als der erstere. Beginnt man nun vorsichtig zu narcotisiren und wendet zunächst die erwähnten Reizstärken an, so fällt zunächst die bei Reizung der unteren Stelle durch centripetale Fortleitung einer sensiblen Erregung erzeugte Unruhe des Körpers weg, während noch Bewegung der Pfote von der oberen und selbstverständlich auch von der unteren Stelle auszulösen ist. Auch bei Anwendung stärkerer Reize gelang es jetzt nicht mehr von der unteren Stelle aus Unruhe des Körpers hervorzurufen. Bei einzelnen Versuchen verschwand bald darnach auch die Pfotenbewegung bei Reizung der oberen Stelle; in vielen Fällen aber musste noch neverlich Aether zugefuihrt werden, um das Wegbleiben derselben zu erzwingen. Fiihrte man jetzt statt Aether feuchte Luft zu, so zeigte sich wieder jedesmal die Reaction der Pforte auf Reizung der oberen Stelle un eine messbare, wenn auch mitnnter kurze Zeit früher als die reflectorischen Bewegungen auf Reizung der untern Stelle. Die Luftzufuhr ist nicht unbedingt erforderlich; sie beschleunigt

1) Der Mechaniker des physiol. Institutes in Prag, Rud. Rothe, verfertigt diese Electroden mit Stativ oder mit Handgriff in verschiedenen Grössen. 
nur das Erwachen ans der Narcose nach Einstellen der Aetherzúfubr.

Da die Reize bei dieser Versuchsanordnung der Qualität nach gleiche waren, und die narcotisirte Stelle fiir die durch einen stärkeren Reiz gesetzte Erregung der sensiblen Fasern in der centripetalen Richtung nflurchgängig, für die durch einen schwächeren Reiz gevetzte Erregung der motorischen Fasern in centrifugaler Richtung noch durchyängig war, so kamn man dieses Ergebniss wohl als einen Beweis a fortiori ansehen und sagen:

Bei localer Narcotisirung des Froschischiadicus mit A ether, Chloroformoder Alcohol erlischtzunachst die Leitungtabigkeit dersensiblen, spiterdiedermotorischen Nervenfasern; beim Erwachen a us der Narcose werden die motorischen Fasern fruber leitungsähig als die sensiblen.

Wir versuchten nun weiter statt der Muskelaction die negative Sohwankmo als Beweis für die Leitumgsähigkeit, respective das Ausbleiben derselben als Beweis fuir die Unterbrechung der Leitungsăhigkeit zu verwenden. Zu diesem Behufe präparirten wir an grossen (ungarischen) Fröschen den Ischiadicus mit seinen Riiekennarkswurzeln frei, legten hieratf an die sensiblen und um die motorischen Wurzeh an ihren Ursprunge je einen Faden und durehsehnitten oberhalb der Unterbindungrsstelle; wurde num noch der Ischiadicus vor seiner Theilung in die Untersehenkeliste durehschnitten, so konnte das Nervenpräparat isolirt herausgehoben werden. Die ganze Präparation wurde mit sorgfültigster Schonung, doch möglichst rasch vorgenommen. Eine Stelle des Ischiadicus wurde nun in die Rinne des Aetherschlauches gelegt; an das perjphere Ende wurden die mit dem Du Bois'schen Schlittenapparat in Verbindung steheuden Platinelectroden gebracht. Jede der Wurzeln wurde iiber ein Paar unpolarisirbarer Thonelectroden gelegt, welche abwechselnd den Galvanometerkreis schlossen. Die eine Electrode wurde mit dem Längsschnitt, die andere mit der Unterbindungsstelle in Berührung gebracht. In den meisten Fällen war der so abgeleitete Nervenstrom und die durch Reizung des Ischiadicus bervirkte negative Schwankung geniigend stark; nur wenn dies nicht der Fall war, wurde die Wurzel mit einem frisch angelegten Querschitt an die Electrode gebracht: hierbei 
waren die durch den Nervenstrom bewirkten Ausschläge oftmals so bedeutende, dass der Compensator angewendet werden musste. Vor der Narcotisirung wurden zunächst die Electroden auf ihren Strom gepriift, bierauf der Strom der motorischen wie der sensiblen Wurzel bestimmt und die Grösse der negativen Sehwankungen der Wurzelströme bei Reizung des Iscliadicus für einen bestimmten Rollenabstand des Inductionsapparates f'estgestellt. Erst jetzt wurde mit der Narcotisirung begonnen. Die Narcose wurde protrahirt, indem wir von der Voranssetzung ausgingen, dass sich in diesem Falle leichter grössere Zeitunterschiede im Eintreten der Lähmung für die beiden Faserarten ergeben könnten. Von dem Momente, in welchem wir mit der Narcotisirung begonnen latten, wurden in möglichst kurzen Zwischenpausen die bei Reizung des Ischiadicus eintretenden Schwankungen der Wurzelströme beobachtet. Nach 3-5 Minuten versehwand jede Spur einer negativen Suhwankung bei Reizung des Ischiadicns und zwar fast regelmässig gleichzeitig für beide Wurzelströme, auch wenn man den Rollenabstand um vieles minderte. Nur ausnahmsweise versebwanden die negativen Sehwankungen der beiden Wurzelströme nicht genau gleichzeitig; der Zeitunterschied betrug immer nur Brnchtheile einer Minute; bald war es der Strom der vorderen, bald der der hinteren Wurzel, für welchen eine negative Schwankung bei Reizung des Ischiadicus etwas länger nachweisbar blieb. Das Wiedererscheinen der negativen Schwankungen nach Luftzufuhr trat ebenfalls für beide Wurzelströme fast regelmässig gleichzeitig ein.

Das Ergebniss dieser Versuche ist keineswegs in voller Uebereinstimmung mit den früheren, da das Sehwinden der negativen Schwankungen fast regelmässig gleichzeitig an beiden Wurzelströmen beobachtet wurde. Wohl ist die negative Schwankung jedesmal Beweis für die Fortleitung der durch deu Reiz gesetzten Erregung bis zur abgeleiteten Stelle, somit in unseren Falle der Beweis für die Leitungsfähigkeit beider Faserarten des Nerven respective Unterbrechung derselben. Doch kann man sich immerbin vorstellen, dass der durch die narcotisirte Stelle von den motorischen Fasern durehgelassene Reiz noch ausreiche, wn eine Bewegung der Extremität hervorzurufen, nicht aber eine merkliche negative Schwankung des Wurzelstromes zul veranlassen; und dies ist um so wahrscheinlicher, als zur Erzeugung einer merklichen negativen Sehwankung in der Regel stärkere Reize nötbig sind, 
als zur Erzengung einer natürlicben Reaction. Diesen stärkeren Reizen entsprechend mussten wir viel länger und stärker narcotisiren, um das Verschwinden der negativen Schwankungen herbeizuführen, als bei den Versuchen, wo wir das Versagen des Reflexes respective das Schwinden der Pfotenzuckungen bei Reizung des Ichiadicus als Beweise für die Unterbrechung der Leitung angesehen hatten.

Der Widerspruch in den Ergebnissen der beiden Versnchsreihen wird gelöst dureh die Annahme, dass wir in der Beobachtung der ebengenannten natürlichen Folge'n der Nervenreizung ein empfindlicheres Reagens auf die Leitungsfäbigkeit der Nervenfasern besitzen als im Verhalten der negativen Schwankung.

Wie bereits eingangs erwähnt wurde, haben wir bei unsern Versuchen ausser Aether woch Chloroform und Alcohol angewendet. Diese Substanzen zeigen deutliche Unterschiede in ihrer Wirkungsweise. Aether wirkt am raschesten, doch verschwindet die Jähmung friiher als bei den beiden anderen Stoffen. In dieser Beziehtung ist nach dem Aether das Chloroform, zum Schlusse Alcohol anzureiben, bei welchem die Lähmung am längsten dauert. Dieses versebiedene Verhalten hängt vielleicht mit der verschieden grossen Fliichtigkeit der drei Mittel zusammen. Auch gelegentlich der allgemeinen Narcose zeigte sich ein erwälnnenswerther Unterschied zwischen Aether und Chloroform. Bringt man einen Frosch in eine Chloroformatmosphäre, so geht er bald durch Herzstillstand zu Grunde. Die motorischen Nervenfasern zeigen keine dentliche Veränderung ihrer Erregl)arkeit, wovon man sich durch Reizung des freigelegten Ischiadicus vor und nach der Chloroformirung iiberzeugen kann. Vor Beginn der Narcotisirung muss der Nerv zwischen die Muskeln gesenkt und die Wunde durch Klemmpincetten gesehlossen werden, um ein directes Einwirken des Chloroforms auf den Nervenstamm zu verhindern. Anders bei einem Frosch, der mit Aether bis zum Erlöschen der Reflexerregbarkeit nareotisirt wurde. Hier haben die motorischen Nerven ihre Erregbarkeit eingehiisst zn einer Zeit, in der die Lebensfahigkeit des Frosches noch soweit erhalten ist, dass er sich nach Entfernung aus der Aetherkammer vollkommen erholen kann.

Zum Schlusse sei noeh kurz erwähnt, dass unsere Versuche einen ganz einwandfreien B.e $w$ e is fi ti $r$ d a s d o p pels in nige Leitungsvermögen der peripheren Nerven ent- 
534 H. Per eles und M. S a ch s: Ueber die Wirkung von Aether etc.

halten. Gelegentlich unserer Wurzelversuche haben wir nicht nur die Wurzeln abwechselnd in den Galvanometerkreis eingeschlossen und den Ischiadicus über Reizelectroden gelegt, sondern auch den Ischiadicusstamm zum Galvanometer abgeleitet und die beiden Wurzeln iuber je ein Paar Reizelectroden gelegt. In allen Fällen rief die Reizung eine negative Schwankung des jeweils abgeleiteten Nervenstroms hervor. Da dieser Erfolg jedesmal aushlieb, wenn der Nerv an irgend einer Stelle seines Verlaufes narcotisirt worden war und nach Luftzufuhr wieder eintrat, so war damit dem Einwande begegnet, dass die negativen Schwankungen der Ausdruck unipolarer Reizungen an den Längsschnitt-Electroden seien 1).

In diesen und ähnlichen Versuchen hat eben die locale Narcotisirung die Beweiskraft der Durchschneidung des Nerven und ihrer Folgen, ist aber dieser gegenüber methodisch werthvoller, weil sie wieder beseitigt werden kann, was für die Durchschneidung nicht gilt.

1) Vergl. Hering, Ueber Schwankungen des Nervenstromes in Folge tnipolarer Reizung. Sitz.-Ber, d. Wiener Ak. 89. Bd. III. Abth. März 1884. 